\title{
Problems in Ophthalmic Drug Delivery: Evaluation of the Interaction Between Preservatives and Cyclodextrins
}

\author{
Bizhan Malaekeh-Nikouei ${ }^{1}$, Bibi Sedigheh Fazly Bazzaz ${ }^{2,{ }^{*}}$, Vahid Soheili ${ }^{3}$, Kobra Mohammadian $^{3}$ \\ ${ }_{1}^{1}$ Nanotechnology Research Centre, School of Pharmacy, Mashhad University of Medical Sciences, Mashhad, IR Iran \\ 2 Biotechnology Research Center, School of Pharmacy, Mashhad University of Medical Sciences, Mashhad, IR Iran \\ 3 Medicinal Chemistry Department, School of Pharmacy, Mashhad University of Medical Sciences, Mashhad, IR Iran \\ ${ }^{*}$ Corresponding author: Bibi Sedigheh Fazly Bazzaz, Biotechnology Research Center; School of Pharmacy, Mashhad University of Medical Sciences, Mashhad, IR Iran. Tel: +98- \\ 5118823252, Fax: +98-5118823251 Email:, E-mail: fazlis@mums.ac.ir.
}

Received: May 14, 2012; Revised: August 07, 2012; Accepted: August 09, 2012

Background: Today, the use of cyclodextrins (CDs) is a major concern in drug formulations to improve solubility and stability of compounds. It has been known that CDs can reduce the antimicrobial effectiveness of preservatives by formation of inclusion complexes. Objectives: In this study the interaction between commonly used preservatives, benzalkonium chloride (BZCl) or methyl paraben (MP), with synthetic CDs (HP $\gamma$-CD or SBE $\beta$-CD) in the presence or absence of ethylene diamine tetra acetic acid (EDTA), as a preservative potentiator, and fluorometholone was investigated.

Materials and Methods: The tests were performed during a 4 week period according to the preservative effectiveness test of USP 2010. Results: The results showed that CDs reduce the effectiveness of $\mathrm{BZCl}$ and $\mathrm{MP}$ even in the presence of drug molecules. The only exception was $\mathrm{HP} \gamma$-CD $5 \%$ solution with BZCl and EDTA which was effective against tested microorganisms both in the presence and absence of drug molecules.

Conclusions: The solution of $\mathrm{HP} \gamma$-CD 5\% with BZCl 0.02\% and EDTA 0.1\% was selected as a good carrier for dissolving fluorometholone for use as an eye drop.

Keywords: Benzalkonium Compounds; Cyclodextrins; Ethylenediaminetetraacetic Acid; Fluorometholone; Parabens

\section{Background}

Cyclodextrins (CDs) are cyclic oligosaccharides with a hydrophilic outer surface and a somewhat hydrophobic central core. The most common natural occurring cyclodextrins are $\alpha$-cyclodextrin ( $\alpha$-CD), $\beta$-cyclodextrin $(\beta$-CD) and $\gamma$-cyclodextrin $(\gamma-C D)$, consisting of six (hexamer), seven (heptamer) and eight (octamer) ( $\alpha-1$, 4)-linked $\alpha$-D-glucopyranose units, respectively (1-7). Many CD derivatives have been synthesized by amination, esterification or etherification of primary and secondary hydroxyl groups of the natural CDs. Depending on the substitution group, the aqueous solubility of CD derivatives are usually different from the natural CDs (1-3, 8).

CDs are capable of interacting with a large variety of guest molecules to form non-covalent inclusion complexes $(3,4,8,9)$ and extensive application of these excipients in pharmaceutical formulations is attributed to their complexation ability. In pharmaceutical products, CDs are used as drug carriers to improve drug dissolution and to enhance drug absorption. Different studies have shown the use of CDs as additives in ophthalmic formulations for increasing the aqueous solubility, aqueous stability and bioavailability of ophthalmic drugs, and to decrease drug irritation (4,10-12). Inclusion in CDs exerts a profound effect on the physicochemical properties of guest molecules as they are temporarily locked or caged within the host cavity but dissociation of drug from CD cavity may occur after dilution, change in ionic state and temperature and competitive displacement $(5,11-14)$. It is believed that the same complexation effect exists with preservatives which are present in pharmaceutical products (15). As only the free preservative molecules are effective, the complexation between preservatives and CDs can reduce their antimicrobial activity $(4,10)$.

\section{Objectives}

The aim of this study was to investigate the interaction of CDs with preservatives in the absence and presence of drug molecules and to show the effect of these interactions on the antimicrobial activity of preservatives. Obviously, the effect of CDs on antimicrobial activity of preservatives depends on their ability to form a

Implication for health policy/practice/research/medical education:

The results of this study are useful in ophthalmic drug delivery because of offering a new formulation in which cyclodexterins simultaneously applied with preservatives. This can be used as a model for other insoluble ophthalmic pharmaceuticals.

Copyright @ C 2013, Ahvaz Jundishapur University of Medical Sciences; Licensee Kowsar Ltd. This is an Open Access article distributed under the terms of the Creative Commons Attribution License (http://creativecommons.org/licenses/by/3.0), which permits unrestricted use, distribution, and reproduction in any medium, provided the original work is properly cited. 
complex with the CD molecule. It is worth to point out that most studies about the effects of CDs on the antimicrobial activity of preservatives were usually in the absence of drug molecules (15-18).

\section{Materials and Methods}

CDs including 2-hydroxypropyl $\gamma$-CD (HP $\gamma$-CD) and sulfobutylether $\beta$-CD sodium salt (SBE $\beta-C D$ ) were purchased from Seebio (China). Preservatives including benzalkonium chloride (BZCl) and methyl paraben (MP) were from Merck (Germany). EDTA was obtained from Kiankaveh pharmaceutical chemicals complex Inc (Iran). Soybean casein digest agar (SCDA) medium was from Himedia (India). $\mathrm{NaCl}$, Tween 80 and lecithin were ordered from Merck (Germany). Fluorometholone was obtained from Pursina pharmaceutical company (Iran).

Aqueous solutions containing 5\%, 10\% and 15\% w/v CD were prepared. Preservatives including $\mathrm{BZCl}(0.01 \% \mathrm{w} / \mathrm{v})$, $\mathrm{MP}(0.2 \%)$ or combination of $\mathrm{BZCl}(0.02 \% \mathrm{w} / \mathrm{v})$ and EDTA $(0.1 \% \mathrm{w} / \mathrm{v})$ were added to these solutions separately ( 19 ). This preparation was repeated with solutions of $\mathrm{HP} \gamma$-CD ( $5 \%$ and $10 \% \mathrm{w} / \mathrm{v})$ containing EDTA $(0.1 \% \mathrm{w} / \mathrm{v})(19), \mathrm{BZCl}$ $(0.02 \% \mathrm{w} / \mathrm{v})(19)$ and fluorometholone $(0.1 \% \mathrm{w} / \mathrm{v})$. These aqueous mixtures were then placed in a rotator (Labinco, Netherlands) for $24 \mathrm{~h}$ at $20 \mathrm{rpm}$. After the equilibration time, solutions were filter sterilized through a $0.22 \mu \mathrm{m}$ sterile filter under aseptic conditions. These solutions were used as samples for carrying out the preservative effectiveness test (PET). PET for each solution was performed according to USP 2010 ( 20 ).

The microorganisms used are listed in Table 1. After cultivation of each microorganism on soybean casein digest agar (SCDA) medium (bacteria kept overnight at $35^{\circ} \mathrm{C}$, Candida albicans at $25^{\circ} \mathrm{C}$ for 2 days, Aspergillus niger at $25^{\circ} \mathrm{C}$ for 1 week), their suspensions in sterile saline $(0.9 \% \mathrm{NaCl})$ or saline-Tween (for A. niger ) were prepared $\left(10^{8} \mathrm{CFU} / \mathrm{mL}\right)$ and vortexed $(10 \mathrm{~s})$ to homogenize. Aliquots $(50 \mu \mathrm{l})$ of these inoculates were added to each 5 $\mathrm{ml}$ sample and were vortexed (10 s). The initial number of each microorganism added to the samples was determined by the plate count method.

The samples were incubated at $25^{\circ} \mathrm{C}$ for 28 days. After 7,14 and 28 days of incubation, the samples were investigated for the number of viable organisms per ml. To do this, $1 \mathrm{ml}$ of each sample was diluted (based on the initially calculated $\mathrm{CFU} / \mathrm{mL}$ ), and transferred to a single sterile plate while SCDA (ca $15 \mathrm{ml}$ at $45^{\circ} \mathrm{C}$ ), containing lecithin $(0.05 \% \mathrm{w} / \mathrm{v})$ and Tween $80(3 \% \mathrm{w} / \mathrm{v})$ as inactivators, were added to each plate. After incubation for 2 days at $35^{\circ} \mathrm{C}$ ( C. albicans for 3 days at $25^{\circ} \mathrm{C}$ and A. niger for 5 days at $25^{\circ} \mathrm{C}$ ) the number of viable organisms per $\mathrm{ml}$ was calculated for each sample by applying the plate count method. Finally, data from HP- $\gamma$ CD 5\% were analyzed by the SPSS software.

\section{Results}

\subsection{First Test}

Table 2 shows the number of microorganisms (CFU) per $\mathrm{ml}$ at the zero time point and 7, 14 and 28 days after incubation of samples in the presence of $\mathrm{BZCl}(0.01 \% \mathrm{w} / \mathrm{v})$ and $\mathrm{HP}$ $\gamma$-CD $(5 \%, 10 \%$, or $15 \% \mathrm{w} / \mathrm{v})$. The results show that, $\mathrm{BZCl}(0.01 \%$ $\mathrm{w} / \mathrm{v}$ ) could not prevent the growth of microorganisms in the presence of different concentrations of HP $\gamma$-CD. Control group results (BZCl $0.01 \% \mathrm{w} / \mathrm{v}$ without $\mathrm{CD}$ ) showed, $\mathrm{BZCl}$ could prevent the growth of microorganisms effectively.

Table 1. Test Strains Used to Investigate the Antimicrobial Effectiveness of Preservatives

\begin{tabular}{lll}
\hline No. & Strain & ATCC \\
\hline $\mathbf{1}$ & Escherichia coli & 8739 \\
$\mathbf{2}$ & Pseudomonas aeruginosa & 9027 \\
$\mathbf{3}$ & Staphylococcus aureus & 6538 \\
$\mathbf{4}$ & Candida albicans & 1021 \\
$\mathbf{5}$ & Aspergillus niger & 16404 \\
\hline
\end{tabular}

Table 2. Number of Viable Counts in Different Formulations at Different Time Intervals

\begin{tabular}{|c|c|c|c|c|}
\hline & $\begin{array}{l}\text { BZCl }+ \text { HP } \\
\gamma \text {-CD 5\% }\end{array}$ & $\begin{array}{l}\text { BZCl + HP } \\
\gamma-\text { CD 10\% }\end{array}$ & $\begin{array}{l}\text { BZCl }+ \text { HP } \\
\gamma-\text { CD 15\% }\end{array}$ & BZCl \\
\hline \multicolumn{5}{|l|}{ Day 0} \\
\hline E. coli & $1.5 \times 10^{6}$ & $1.5 \times 10^{6}$ & $1.5 \times 10^{6}$ & $1.5 \times 10^{6}$ \\
\hline $\begin{array}{l}\text { P. aerugi- } \\
\text { nosa }\end{array}$ & $10^{6}$ & $10^{6}$ & $10^{6}$ & $10^{6}$ \\
\hline S.aureus & $5 \times 10^{5}$ & $5 \times 10^{5}$ & $5 \times 10^{5}$ & $5 \times 10^{5}$ \\
\hline C. albicans & $10^{5}$ & $10^{5}$ & $10^{5}$ & $10^{5}$ \\
\hline A. niger & $2 \times 10^{4}$ & $2 \times 10^{4}$ & $2 \times 10^{4}$ & $2 \times 10^{4}$ \\
\hline \multicolumn{5}{|l|}{ Day 7th } \\
\hline E. coli & $6 \times 10^{6}$ & $2 \times 10^{6}$ & $6 \times 10^{7}$ & $\leq 9 \times 10^{2}$ \\
\hline $\begin{array}{l}\text { P. aerugi- } \\
\text { nosa }\end{array}$ & $5 \times 10^{6}$ & $2 \times 10^{6}$ & $1.5 \times 10^{7}$ & $\leq 9 \times 10^{2}$ \\
\hline S.aureus & $\leq 9 \times 10^{3}$ & $\leq 9 \times 10^{3}$ & $4 \times 10^{4}$ & $\leq 9 \times 10^{2}$ \\
\hline C. albicans & $3 \times 10^{5}$ & $6 \times 10^{6}$ & $6 \times 10^{6}$ & $\leq 9 \times 10^{2}$ \\
\hline A. niger & $10^{5}$ & $5 \times 10^{5}$ & $4 \times 10^{5}$ & $\leq 9 \times 10^{2}$ \\
\hline \multicolumn{5}{|l|}{ Day 14th } \\
\hline E. coli & $8 \times 10^{6}$ & $2 \times 10^{6}$ & $5 \times 10^{6}$ & $\leq 9$ \\
\hline $\begin{array}{l}\text { P. aerugi- } \\
\text { nosa }\end{array}$ & $6 \times 10^{5}$ & $4 \times 10^{6}$ & $1.5 \times 10^{7}$ & $\leq 9$ \\
\hline S. aureus & $\leq 9 \times 10$ & $\leq 9 \times 10$ & $3 \times 10^{3}$ & $\leq 9$ \\
\hline C. albicans & $6 \times 10^{5}$ & $3 \times 10^{5}$ & $3 \times 10^{6}$ & $\leq 9$ \\
\hline A. niger & $9 \times 10^{4}$ & $10^{5}$ & $8 \times 10^{4}$ & $\leq 9$ \\
\hline \multicolumn{5}{|l|}{ Day 28th } \\
\hline E. coli & $6 \times 10^{6}$ & $5 \times 10^{6}$ & $4 \times 10^{6}$ & 0 \\
\hline $\begin{array}{l}\text { P. aerugi- } \\
\text { nosa }\end{array}$ & $4 \times 10^{5}$ & $5 \times 10^{6}$ & $4 \times 10^{6}$ & 0 \\
\hline S. aureus & 0 & 0 & $\leq 9 \times 10$ & 0 \\
\hline C.albicans & $9 \times 10^{5}$ & $1.2 \times 10^{6}$ & $4 \times 10^{6}$ & 0 \\
\hline A. niger & $9 \times 10^{4}$ & $8 \times 10^{5}$ & $10^{5}$ & 0 \\
\hline
\end{tabular}




\subsection{Second Test}

Table 3 shows the number of microorganisms (CFU) per $\mathrm{ml}$ at the zero time and 7, 14 and 28 days after incubation of samples in the presence of BZCl $(0.01 \% \mathrm{w} / \mathrm{v})$ and SBE $\beta-C D(5 \%, 10 \%$, or $15 \% \mathrm{w} / \mathrm{v})$. The results show that, $\mathrm{BZCl}(0.01 \% \mathrm{w} / \mathrm{v})$ could not prevent the growth of microorganisms in the presence of all of the concentrations of SBE $\beta$-CD. The control group ( $\mathrm{BZCl} 0.01 \% \mathrm{w} / \mathrm{v}$ without CD) showed that $\mathrm{BZCl}$ could prevent the growth of microorganisms effectively.

Table 3. Number of Viable Counts in Different Formulations at Different Time Intervals

\begin{tabular}{|c|c|c|c|c|}
\hline & $\begin{array}{l}\text { BZCl + } \\
\text { SBE } \beta-C D \\
5 \%\end{array}$ & $\begin{array}{l}\text { BZCl + } \\
\text { SBE } \beta-C D \\
10 \%\end{array}$ & $\begin{array}{l}\text { BZCl + } \\
\text { SBE } \beta-C D \\
15 \%\end{array}$ & BZCl \\
\hline \multicolumn{5}{|l|}{ Day 0} \\
\hline E. coli & $10^{7}$ & $10^{7}$ & $10^{7}$ & $10^{7}$ \\
\hline $\begin{array}{l}\text { P. aerugi- } \\
\text { nosa }\end{array}$ & $1.2 \times 10^{6}$ & $1.2 \times 10^{6}$ & $1.2 \times 10^{6}$ & $1.2 \times 10^{6}$ \\
\hline S. aureus & $10^{6}$ & $10^{6}$ & $10^{6}$ & $10^{6}$ \\
\hline C. albicans & $1.2 \times 10^{5}$ & $1.2 \times 10^{5}$ & $1.2 \times 10^{5}$ & $1.2 \times 10^{5}$ \\
\hline A. niger & $10^{5}$ & $10^{5}$ & $10^{5}$ & $10^{5}$ \\
\hline \multicolumn{5}{|l|}{ Day 7th } \\
\hline E. coli & $8 \times 10^{7}$ & $4 \times 10^{7}$ & $2 \times 10^{4}$ & $\leq 9 \times 10^{2}$ \\
\hline $\begin{array}{l}\text { P. aerugi- } \\
\text { nosa }\end{array}$ & $1.2 \times 10^{7}$ & $9 \times 10^{7}$ & $1.2 \times 10^{9}$ & $\leq 9 \times 10^{2}$ \\
\hline S. aureus & $\leq 9 \times 10^{2}$ & $2 \times 10^{4}$ & $\leq 9 \times 10^{2}$ & $\leq 9 \times 10^{2}$ \\
\hline C. albicans & $3 \times 10^{5}$ & $8 \times 10^{4}$ & $8 \times 10^{5}$ & $\leq 9 \times 10^{2}$ \\
\hline A. niger & $10^{5}$ & $10^{6}$ & $10^{7}$ & $\leq 9 \times 10^{2}$ \\
\hline \multicolumn{5}{|l|}{ Day 14th } \\
\hline E. coli & $6 \times 10^{7}$ & $2 \times 10^{7}$ & $1.4 \times 10^{8}$ & $\leq 9$ \\
\hline $\begin{array}{l}\text { P. aerugi- } \\
\text { nosa }\end{array}$ & $6 \times 10^{7}$ & $2 \times 10^{7}$ & $2 \times 10^{8}$ & $\leq 9$ \\
\hline S. aureus & $\leq 9 \times 10$ & $\leq 9 \times 10$ & $\leq 9 \times 10$ & $\leq 9$ \\
\hline C. albicans & $3 \times 10^{5}$ & $10^{6}$ & $6 \times 10^{6}$ & $\leq 9$ \\
\hline A. niger & $3 \times 10^{5}$ & $10^{6}$ & $5 \times 10^{6}$ & $\leq 9 \times 10^{2}$ \\
\hline \multicolumn{5}{|l|}{ Day 28th } \\
\hline E. coli & $5 \times 10^{7}$ & $2 \times 10^{7}$ & $1.3 \times 10^{8}$ & 0 \\
\hline $\begin{array}{l}\text { P. aerugi- } \\
\text { nosa }\end{array}$ & $3 \times 10^{7}$ & $3 \times 10^{7}$ & $1.2 \times 10^{8}$ & 0 \\
\hline S. aureus & 0 & 0 & 0 & 0 \\
\hline C. albicans & $6 \times 10^{5}$ & $4 \times 10^{6}$ & $2 \times 10^{7}$ & 0 \\
\hline A. niger & $5 \times 10^{5}$ & $2 \times 10^{6}$ & $6 \times 10^{6}$ & 0 \\
\hline
\end{tabular}

\subsection{Third Test}

Table 4 shows the number of microorganisms (CFU) per $\mathrm{ml}$ at the zero time point and 7,14 and 28 days after incubation of samples in the presence of MP $(0.2 \% \mathrm{w} / \mathrm{v})$ and $\mathrm{HP}$
$\gamma$-CD ( $5 \%, 10 \%$, or $15 \% \mathrm{w} / \mathrm{v})$. The results show that, MP (0.2\%) could not prevent the growth of microorganisms in the presence of different concentrations of HP $\gamma$-CD. The control group (MP $0.2 \% \mathrm{w} / \mathrm{v}$ without $\mathrm{CD}$ ) showed, MP could prevent the growth of microorganisms effectively.

Table 4. Number of Viable Counts in Different Formulations at Different Time Intervals

\begin{tabular}{|c|c|c|c|c|}
\hline & $\begin{array}{l}\text { MP + HP } \\
\gamma-\text { CD 5\% }\end{array}$ & $\begin{array}{l}\text { MP + HP } \\
\gamma-\text { CD 10 } \%\end{array}$ & $\begin{array}{l}\mathrm{MP}+\mathrm{HP} \\
\gamma-\mathrm{CD} 15 \%\end{array}$ & MP \\
\hline \multicolumn{5}{|l|}{ Day 0} \\
\hline E. coli & $4 \times 10^{5}$ & $4 \times 10^{5}$ & $4 \times 10^{5}$ & $4 \times 10^{5}$ \\
\hline $\begin{array}{l}\text { P. aerugi- } \\
\text { nosa }\end{array}$ & $4 \times 10^{5}$ & $4 \times 10^{5}$ & $4 \times 10^{5}$ & $4 \times 10^{5}$ \\
\hline S. aureus & $7 \times 10^{5}$ & $7 \times 10^{5}$ & $7 \times 10^{5}$ & $7 \times 10^{5}$ \\
\hline C. albicans & $3 \times 10^{4}$ & $3 \times 10^{4}$ & $3 \times 10^{4}$ & $3 \times 10^{4}$ \\
\hline A. niger & $8 \times 10^{4}$ & $8 \times 10^{4}$ & $8 \times 10^{4}$ & $8 \times 10^{4}$ \\
\hline \multicolumn{5}{|l|}{ Day 7th } \\
\hline E. coli & $1.2 \times 10^{8}$ & $4 \times 10^{7}$ & $6 \times 10^{7}$ & $\leq 9 \times 10^{2}$ \\
\hline $\begin{array}{l}\text { P. aerugi- } \\
\text { nosa }\end{array}$ & $5 \times 10^{7}$ & $1.5 \times 10^{6}$ & $3 \times 10^{7}$ & $\leq 9 \times 10^{2}$ \\
\hline S. aureus & $4 \times 10^{6}$ & $4 \times 10^{5}$ & $2 \times 10^{5}$ & $\leq 9 \times 10^{2}$ \\
\hline C. albicans & $7 \times 10^{5}$ & $10^{7}$ & $7 \times 10^{5}$ & $\leq 9 \times 10^{2}$ \\
\hline A. niger & $10^{5}$ & $10^{6}$ & $8 \times 10^{5}$ & $\leq 9 \times 10^{2}$ \\
\hline \multicolumn{5}{|l|}{ Day 14th } \\
\hline E. coli & $1.2 \times 10^{8}$ & $1.4 \times 10^{7}$ & $8 \times 10^{7}$ & $\leq 9$ \\
\hline $\begin{array}{l}\text { P. aerugi- } \\
\text { nosa }\end{array}$ & $10^{8}$ & $1.3 \times 10^{6}$ & $1.5 \times 10^{7}$ & $\leq 9$ \\
\hline S. aureus & $1.8 \times 10^{5}$ & $1.3 \times 10^{5}$ & $5 \times 10^{4}$ & $\leq 9$ \\
\hline C. albicans & $1.4 \times 10^{6}$ & $1.2 \times 10^{6}$ & $1.2 \times 10^{6}$ & $\leq 9$ \\
\hline A. niger & $1.5 \times 10^{5}$ & $1.3 \times 10^{6}$ & $1.8 \times 10^{6}$ & $\leq 9$ \\
\hline \multicolumn{5}{|l|}{ Day 28th } \\
\hline E. coli & $3 \times 10^{7}$ & $1.6 \times 10^{7}$ & $5 \times 10^{7}$ & 0 \\
\hline $\begin{array}{l}\text { P. aerugi- } \\
\text { nosa }\end{array}$ & $1.2 \times 10^{8}$ & $9 \times 10^{5}$ & $1.8 \times 10^{7}$ & 0 \\
\hline S. aureus & $5 \times 10^{4}$ & $2 \times 10^{4}$ & $4 \times 10^{4}$ & 0 \\
\hline C. albicans & $5 \times 10^{5}$ & $4 \times 10^{5}$ & $1.2 \times 10^{6}$ & 0 \\
\hline A. niger & $10^{5}$ & $1.3 \times 10^{6}$ & $10^{6}$ & 0 \\
\hline
\end{tabular}

\subsection{Forth Test}

Table 5 shows the number of microorganisms (CFU) per $\mathrm{ml}$ at the zero time point and 7,14 and 28 days after incubation of samples in the presence of MP $(0.2 \% \mathrm{w} / \mathrm{v})$ and $\operatorname{SBE} \beta-\mathrm{CD}(5 \%, 10 \%$, or $15 \% \mathrm{w} / \mathrm{v})$. The results show that, MP $(0.2 \% \mathrm{w} / \mathrm{v})$ could not prevent the growth of microorganisms in the presence of different concentrations of SBE $\beta$-CD. The control group (MP $0.2 \% \mathrm{w} / \mathrm{v}$ without CD) showed, MP could prevent the growth of microorganisms effectively. 
Table 5. Number of Viable Counts in Different Formulations at Different Time Intervals

\begin{tabular}{|c|c|c|c|c|}
\hline & $\begin{array}{l}\text { MP + SBE } \\
\beta \text {-CD 5\% }\end{array}$ & $\begin{array}{l}\text { MP + SBE } \\
\beta-C D 10 \% \\
\end{array}$ & $\begin{array}{l}\text { MP + SBE } \\
\beta-C D 15 \%\end{array}$ & MP \\
\hline \multicolumn{5}{|l|}{ Day 0} \\
\hline E. coli & $1.9 \times 10^{5}$ & $1.9 \times 10^{5}$ & $1.9 \times 10^{5}$ & $1.9 \times 10^{5}$ \\
\hline $\begin{array}{l}\text { P. aerugi- } \\
\text { nosa }\end{array}$ & $5 \times 10^{5}$ & $5 \times 10^{5}$ & $5 \times 10^{5}$ & $5 \times 10^{5}$ \\
\hline S.aureus & $1.7 \times 10^{5}$ & $1.7 \times 10^{5}$ & $1.7 \times 10^{5}$ & $1.7 \times 10^{5}$ \\
\hline C. albicans & $5 \times 10^{4}$ & $5 \times 10^{4}$ & $5 \times 10^{4}$ & $5 \times 10^{4}$ \\
\hline A. niger & $10^{5}$ & $10^{5}$ & $10^{5}$ & $10^{5}$ \\
\hline \multicolumn{5}{|l|}{ Day 7th } \\
\hline E. coli & $3 \times 10^{7}$ & $5 \times 10^{7}$ & $1.4 \times 10^{8}$ & $\leq 9 \times 10^{2}$ \\
\hline $\begin{array}{l}\text { P. aerugi- } \\
\text { nosa }\end{array}$ & $10^{8}$ & $4 \times 10^{7}$ & $10^{9}$ & $\leq 9 \times 10^{2}$ \\
\hline S.aureus & $1.3 \times 10^{6}$ & $6 \times 10^{7}$ & $4 \times 10^{5}$ & $\leq 9 \times 10^{2}$ \\
\hline C. albicans & $4 \times 10^{5}$ & $3 \times 10^{6}$ & $4 \times 10^{6}$ & $\leq 9 \times 10^{2}$ \\
\hline A. niger & $3 \times 10^{5}$ & $7 \times 10^{5}$ & $4 \times 10^{5}$ & $\leq 9 \times 10^{2}$ \\
\hline \multicolumn{5}{|l|}{ Day 14th } \\
\hline E. coli & $3 \times 10^{7}$ & $6 \times 10^{7}$ & $10^{8}$ & $\leq 9$ \\
\hline $\begin{array}{l}\text { P. aerugi- } \\
\text { nosa }\end{array}$ & $6 \times 10^{6}$ & $4 \times 10^{6}$ & $6 \times 10^{7}$ & $\leq 9$ \\
\hline S. aureus & $9 \times 10^{4}$ & $1.6 \times 10^{5}$ & $6 \times 10^{4}$ & $\leq 9$ \\
\hline C. albicans & $4 \times 10^{5}$ & $2 \times 10^{6}$ & $2 \times 10^{6}$ & $\leq 9$ \\
\hline A. niger & $4 \times 10^{5}$ & $8 \times 10^{5}$ & $10^{6}$ & $\leq 9$ \\
\hline \multicolumn{5}{|l|}{ Day 28th } \\
\hline E. coli & $1.2 \times 10^{8}$ & $5 \times 10^{7}$ & $9 \times 10^{7}$ & 0 \\
\hline $\begin{array}{l}\text { P. aerugi- } \\
\text { nosa }\end{array}$ & $1.7 \times 10^{7}$ & $5 \times 10^{6}$ & $4 \times 10^{7}$ & 0 \\
\hline S. aureus & $3 \times 10^{6}$ & $3 \times 10^{3}$ & $2 \times 10^{3}$ & 0 \\
\hline C. albicans & $9 \times 10^{5}$ & $2 \times 10^{6}$ & $3 \times 10^{6}$ & 0 \\
\hline A. niger & $7 \times 10^{5}$ & $10^{6}$ & $1.1 \times 10^{6}$ & 0 \\
\hline
\end{tabular}

\subsection{Fifth Test}

To ensure whether the preservatives-CD system in the presence of drug molecules can prevent the growth of microorganisms effectively, the tests with fluorometholone $(0.1 \% \mathrm{w} / \mathrm{v})$ and SBE $\beta$-CD ( $5 \%$ or $15 \% \mathrm{w} / \mathrm{v})$ complexes were repeated in the presence of either $\mathrm{BZCl}(0.01 \% \mathrm{w} / \mathrm{v})$ or MP $(0.1 \% \mathrm{w} / \mathrm{v})$, but the preservatives did not show enough effectiveness against the microorganisms tested (data are not shown). This test was also repeated with $\operatorname{BZCl}(0.02 \% \mathrm{w} / \mathrm{v})$ and MP $(0.2 \% \mathrm{w} / \mathrm{v})$ together for both cyclodexterins (SBE $\beta$-CD and HP $\gamma$-CD but without drug), but they couldn't inhibit the growth of the tested microorganisms (data are not shown). So EDTA (0.1\% w/v), as a preservative potentiator, was replaced instead of MP and the test was carried out for 28 days.
Table 6. Number of Viable Counts in Different Formulations at Different Time Intervals

\begin{tabular}{|c|c|c|c|c|c|c|}
\hline & $\begin{array}{l}\text { EDTA } \\
+ \text { BZCl } \\
+ \text { HP } \\
\gamma-\text { CD } \\
5 \%\end{array}$ & $\begin{array}{l}\text { EDTA } \\
+ \text { BZCl } \\
+ \text { HP } \\
\gamma \text {-CD } \\
10 \%\end{array}$ & $\begin{array}{l}\text { EDTA } \\
+ \text { BZCl } \\
+ \text { HP } \\
\gamma-\text { CD } \\
\mathbf{1 5} \%\end{array}$ & $\begin{array}{l}\text { EDTA } \\
+ \text { BZCl } \\
+ \text { SBE } \\
\beta-C D \\
5 \%\end{array}$ & $\begin{array}{l}\text { EDTA } \\
+ \text { BZCl } \\
+ \text { SBE } \\
\beta-C D \\
10 \%\end{array}$ & $\begin{array}{l}\text { DTA + } \\
\text { BZCl } \\
+ \text { SBE } \\
\beta-C D \\
15 \%\end{array}$ \\
\hline \multicolumn{7}{|l|}{ Day 0} \\
\hline E. coli & $\begin{array}{l}8.3 \times \\
10^{5}\end{array}$ & $\begin{array}{l}8.3 \times \\
10^{5}\end{array}$ & $\begin{array}{l}8.3 \times \\
10^{5}\end{array}$ & $\begin{array}{l}8.3 \times \\
10^{5}\end{array}$ & $\begin{array}{l}8.3 \times \\
10^{5}\end{array}$ & $\begin{array}{l}8.3 \times \\
10^{5}\end{array}$ \\
\hline $\begin{array}{l}\text { P. aeru- } \\
\text { ginosa }\end{array}$ & $\begin{array}{l}8.3 \times \\
10^{5}\end{array}$ & $\begin{array}{l}8.3 \times \\
10^{5}\end{array}$ & $\begin{array}{l}8.3 \times \\
10^{5}\end{array}$ & $\begin{array}{l}8.3 \times \\
10^{5}\end{array}$ & $\begin{array}{l}8.3 \times \\
10^{5}\end{array}$ & $\begin{array}{l}8.3 \times \\
10^{5}\end{array}$ \\
\hline $\begin{array}{l}\text { S. au- } \\
\text { reus }\end{array}$ & $\begin{array}{l}2.5 \times \\
10^{5}\end{array}$ & $\begin{array}{l}2.5 \times \\
10^{5}\end{array}$ & $\begin{array}{l}2.5 \times \\
10^{5}\end{array}$ & $\begin{array}{l}2.5 \times \\
10^{5}\end{array}$ & $\begin{array}{l}2.5 \times \\
10^{5}\end{array}$ & $\begin{array}{l}2.5 \times \\
10^{5}\end{array}$ \\
\hline $\begin{array}{l}\text { C. albi- } \\
\text { cans }\end{array}$ & $\begin{array}{l}2.5 \times \\
10^{4}\end{array}$ & $\begin{array}{l}2.5 \times \\
10^{4}\end{array}$ & $\begin{array}{l}2.5 \times \\
10^{4}\end{array}$ & $\begin{array}{l}2.5 \times \\
10^{4}\end{array}$ & $\begin{array}{l}2.5 \times \\
10^{4}\end{array}$ & $\begin{array}{l}2.5 \times \\
10^{4}\end{array}$ \\
\hline $\begin{array}{l}\text { A. } \\
\text { niger }\end{array}$ & $\begin{array}{l}2.8 \times \\
10^{5}\end{array}$ & $\begin{array}{l}2.8 \times \\
10^{5}\end{array}$ & $\begin{array}{l}2.8 \times \\
10^{5}\end{array}$ & $\begin{array}{l}2.8 \times \\
10^{5}\end{array}$ & $\begin{array}{l}2.8 \times \\
10^{5}\end{array}$ & $\begin{array}{l}2.8 \times \\
10^{5}\end{array}$ \\
\hline \multicolumn{7}{|c|}{ Day 7th } \\
\hline E. coli & 0 & 0 & $\begin{array}{l}1.2 \times \\
10^{6}\end{array}$ & $\begin{array}{l}1.7 \times \\
10^{6}\end{array}$ & $7 \times 10^{6}$ & $9 \times 10^{7}$ \\
\hline $\begin{array}{l}\text { P. aeru- } \\
\text { ginosa }\end{array}$ & 0 & $4 \times 10^{5}$ & $\begin{array}{l}1.6 \times \\
10^{7}\end{array}$ & $\begin{array}{l}2.7 \times \\
10^{7}\end{array}$ & $3 \times 10^{7}$ & $\begin{array}{l}3.2 \times \\
10^{6}\end{array}$ \\
\hline $\begin{array}{l}\text { S. au- } \\
\text { reus }\end{array}$ & 0 & 0 & 0 & $5 \times 10^{7}$ & $\begin{array}{l}1.2 \times \\
10^{7}\end{array}$ & 0 \\
\hline $\begin{array}{l}\text { C. albi- } \\
\text { cans }\end{array}$ & 0 & 0 & $5 \times 10^{5}$ & $\begin{array}{l}2.5 \times \\
10^{5}\end{array}$ & $\begin{array}{l}1.7 \times \\
10^{5}\end{array}$ & 0 \\
\hline $\begin{array}{l}\text { A. } \\
\text { niger }\end{array}$ & 0 & 0 & $\begin{array}{l}2.5 \times \\
10^{6}\end{array}$ & $\begin{array}{l}2.3 \times \\
10^{6}\end{array}$ & $\begin{array}{l}2.6 \times \\
10^{6}\end{array}$ & $\begin{array}{l}2.6 \times \\
10^{5}\end{array}$ \\
\hline \multicolumn{7}{|c|}{ Day 14th } \\
\hline E. coli & 0 & 0 & - & - & - & - \\
\hline $\begin{array}{l}\text { P. aeru- } \\
\text { ginosa }\end{array}$ & 0 & $10^{5}$ & - & - & - & - \\
\hline $\begin{array}{l}\text { S. au- } \\
\text { reus }\end{array}$ & 0 & 0 & - & - & - & - \\
\hline $\begin{array}{l}\text { C. albi- } \\
\text { cans }\end{array}$ & 0 & 0 & - & - & - & - \\
\hline $\begin{array}{l}\text { A. } \\
\text { niger }\end{array}$ & 0 & 0 & - & - & - & - \\
\hline \multicolumn{7}{|c|}{ Day 28th } \\
\hline E. coli & 0 & - & - & - & - & - \\
\hline $\begin{array}{l}\text { P. aeru- } \\
\text { ginosa }\end{array}$ & 0 & - & - & - & - & - \\
\hline $\begin{array}{l}\text { S.au- } \\
\text { reus }\end{array}$ & 0 & - & - & - & - & - \\
\hline $\begin{array}{l}\text { C. albi- } \\
\text { cans }\end{array}$ & 0 & - & - & - & - & - \\
\hline $\begin{array}{l}\text { A. } \\
\text { niger }\end{array}$ & 0 & - & - & - & - & - \\
\hline
\end{tabular}




\subsection{Sixth Test}

\subsubsection{First Week}

Table 6 shows the number of microorganisms (CFU) per $\mathrm{ml}$ at the zero time point and 7, 14 and 28 days after incubation of samples in the presence of EDTA $(0.1 \% \mathrm{w} / \mathrm{v})$, $\operatorname{BZCl}(0.02 \% \mathrm{w} / \mathrm{v})$ and either HP $\gamma$-CD or SBE- $\beta(5 \%, 10 \%$, or $15 \% \mathrm{w} / \mathrm{v})$. The results show that, EDTA $(0.1 \% \mathrm{w} / \mathrm{v})$ and BZCl $(0.02 \% \mathrm{w} / \mathrm{v})$ could not prevent the growth of microorganisms in the presence of $\mathrm{HP} \gamma$-CD $15 \% \mathrm{w} / \mathrm{v}$ and SBE- $\beta(5 \%, 10 \%$, or $15 \% \mathrm{w} / \mathrm{v}$ ) but they could prevent the growth of microorganisms effectively in the presence of $\mathrm{HP} \gamma$-CD 5\% w/v. In the presence of $\mathrm{HP} \gamma$-CD $10 \% \mathrm{w} / \mathrm{v}$, only Pseudomonas. aeruginosa could grow, so the study was continued to see if this could be prevented.

\subsubsection{Second Week}

After 14 days, the results confirmed that EDTA $(0.1 \% \mathrm{w} / \mathrm{v})$ and $\mathrm{BZCl}(0.02 \% \mathrm{w} / \mathrm{v})$ could prevent the growth of microorganisms in the presence of HP $\gamma$-CD 5\% but they could not prevent the growth of $P$. aeruginosa in the presence of $\mathrm{HP} \gamma$-CD $10 \%$. So the study was continued only with HP $\gamma$-CD 5\% w/v.

\subsubsection{Fourth Week}

As shown in Table 6, in the presence of EDTA $(0.1 \% \mathrm{w} / \mathrm{v})$, $\operatorname{BZCl}(0.02 \% \mathrm{w} / \mathrm{v})$ and $\mathrm{HP} \gamma-\mathrm{CD} 5 \% \mathrm{w} / \mathrm{v}$, the growth of all tested microorganisms were fully prevented.

Table 7. Number of Viable Counts in Different Formulations at Different Time Intervals

\begin{tabular}{|c|c|c|c|c|}
\hline & Day 0 & Day $7^{\text {th }}$ & Day $14^{\text {th }}$ & Day $28^{\text {th }}$ \\
\hline \multicolumn{5}{|c|}{ EDTA + BZCI + HP $\gamma$-CD 5\% + drug } \\
\hline E. coli & $1.8 \times 10^{9}$ & 0 & 0 & 0 \\
\hline P. aeruginosa & $4.6 \times 10^{6}$ & $3.1 \times 10^{4}$ & 0 & 0 \\
\hline S. aureus & $6.4 \times 10^{7}$ & 0 & 0 & 0 \\
\hline C. albicans & $7.3 \times 10^{7}$ & 0 & 0 & 0 \\
\hline A. niger & $2.7 \times 10^{7}$ & 0 & 0 & 0 \\
\hline \multicolumn{5}{|c|}{ EDTA + BZCl + HP $\gamma-$ CD 10 $\%+$ drug } \\
\hline E. coli & $1.8 \times 10^{9}$ & $4.2 \times 10^{8}$ & - & - \\
\hline P. aeruginosa & $4.6 \times 10^{6}$ & $6 \times 10^{7}$ & - & - \\
\hline S. aureus & $6.4 \times 10^{7}$ & $1.1 \times 10^{6}$ & - & - \\
\hline C. albicans & $7.3 \times 10^{7}$ & $1.8 \times 10^{5}$ & - & - \\
\hline A. niger & $2.7 \times 10^{7}$ & $2 \times 10^{5}$ & - & - \\
\hline
\end{tabular}

\subsection{Seventh Test}

According to these data, HP $\gamma$-CD 5\% w/v was chosen for dissolving fluorometholone. To ensure the effectiveness of these preservatives, the test was repeated in the presence of fluorometholone. The data are shown in Table 7. Once again, the preservatives could not prevent the growth of P. aeroginosa in the presence of $\operatorname{HP} \gamma$-CD $10 \%$. So we focused on using the HP- $\gamma$ 5\% w/v and continued the study only with this concentration. The results show that, EDTA $(0.1 \% \mathrm{w} / \mathrm{v})$ and $\mathrm{BZCl}(0.02 \% \mathrm{w} / \mathrm{v})$ could prevent the growth of microorganisms in the presence of $\mathrm{HP} \gamma$-CD $5 \%$ w/v and fluorometholone.

\subsection{Statistical Analysis of Data}

The resulting data of HP- $\gamma$ CD $5 \%$ were also analyzed by univariate analysis of variance of the SPSS software. Univariate analysis of variance tests the null hypotheses about the effects of other variables on the means of different groups of a dependent variable. Moreover, it investigates the interactions between factors as well as the influence of individual factors. Accordingly formulation and strain of microorganisms have an influence on the logarithm of the mean number of viable counts (log) (sig $<0.05$ ). Also, different formulations inoculated by various microorganisms, different formulations from various days, different microorganisms from various days, and different formulations inoculated by various microorganisms on each day all have an influence on the logarithm of the mean number of viable counts ( $\operatorname{sig}<0.05$ ).

As an overall F test showed significance, Post hoc tests were used to evaluate differences among means of viable counts of formulations. Thus Tukey test of Post hoc multiple comparisons was used to evaluate if there is a significant difference between various formulations. According to the results, a significant difference between various formulations was observed at the 0.05 level. These differences are remarkable enough to categorize formulations in contrast to the number of viable counts. Moreover, formulation $\mathrm{EDTA}+\mathrm{BZCl}+\mathrm{HP} \gamma-\mathrm{CD} 5 \%$ has the best effects on the microorganisms.

Tukey test was also used to determine if there are significant differences between one microorganism and the others in terms of response to preservatives. The results show that there is a significant difference between Gram positive bacteria (Staphylococcus aureus), Gram negative bacteria ( $P$. aeroginosa) and fungi (A. niger and C. albicans) in their susceptibility to formulations and S. aureus is more susceptible than the others. Because of various structures of cell wall, these microorganisms showed different mechanisms against the applied preservatives. However it should be noted the response of A. niger is somewhat resemble to $S$. aureus.

\section{Discussion}

CDs can form inclusion complexes with many compounds through their cavities, which enable them to take up whole or some parts of a molecule. This ability makes 
CDs appropriate for encapsulation techniques as it alters many physicochemical properties of molecules such as solubility, stability against light, heat, oxidizing conditions, and volatility of compounds $(12,13,16)$. These properties have expanded applications of CDs in food $(2,21)$, pharmaceuticals $(2,22,23)$, agricultures and chromatographic techniques (2), and environmental technologies (24).

Since fluorometholone ophthalmic drop is in the form of suspension and this can lead to many problems (e.g. non homogenous dispersion of drug, aggregation and increased production cost), we decided to prepare a solution form by using CDs. Obviously, aqueous solutions of cyclodextrins are in the risk of microbial contamination, so addition of preservatives is necessary (16). Many studies have previously investigated the interaction of CDs and preservatives $(2,15,17$, $18,25)$. Chan et al. (15) investigated the interaction of $\beta$-cyclodextrin and parabens in aqueous solutions. After preparation of solutions through various processes, the interactions were evaluated through dialysis membranes. Their study showed that the interaction between preservatives and CDs increased with increased concentration of CDs, and this interaction was not influenced by the means of preparation. They also showed the interaction between $C D$ and preservative was under the effect of size and lipophilic properties of preservative molecules, and the highest interaction was with MP due to it's small size.

In addition, the $\beta$-CD inclusion complex with sorbic acid was characterized by nuclear magnetic resonance (NMR) spectroscopy in the study by Zhang et al. (25). The interactions between several commonly used preservatives and 2-hydroxypropyl- $\beta$-CD were investigated by Loftsson et al. They showed that the antimicrobial effectiveness of lipophilic preservatives were more affected by CDs (18). Since only free preservative molecules are effective against microorganisms $(4,16)$, choosing the appropriate preservative for complicated drug formulations is sometimes difficult and modifications are required for certain formulations. Furthermore, the use of high concentrations of preservatives may have adverse effects for consumers (16). It appears that the effectiveness of preservatives depends on their degree of complexation (18).

The effects of CDs on the antimicrobial activity of preservatives have commonly been studied in the absence of drug molecules ( $15-18$ ). We assumed that the presence of drug molecules in the complex form may reduce the interaction of CDs and preservatives. So, this interaction was studied in the presence of fluorometholone molecules. As the results showed HP- $\gamma$ and SBE- $\beta$ CDs have reduced the effectiveness of $\mathrm{BZCl}$ and MP in the presence or absence of EDTA (Table 2 -5). This effect was intensified when $\mathrm{CD}$ concentration was increased. It seemed that in the presence of drug molecules, effective amounts of bounded preservatives was not released as there was no reduction in the number of $P$. aeruginosa in the HP- $\gamma 10 \% \mathrm{w} / \mathrm{v}$ solution (Table 7 ).

In fact, the affinity of preservative molecules to form complexes with CDs may be greater than that of drug molecules. Therefore, addition of preservatives to CDs results in the formation of inclusion complexes by hydrophobic cavity of CDs and decrease of preservative effectiveness. The only exception was the solution of HP$\gamma$-CD 5\% w/v, which represented excellent results in inhibition of microorganisms in the presence or absence of drug molecules (Table 6 and 7). The effects of different formulations are shown in Figure $1-4$. Obviously the suggested compounds (EDTA + BZCl + HP $\gamma$-CD 5\%) are the most suitable preservatives in combination with HP $\gamma$-CD 5\% for preparation of fluorometholone solution as eye drops.

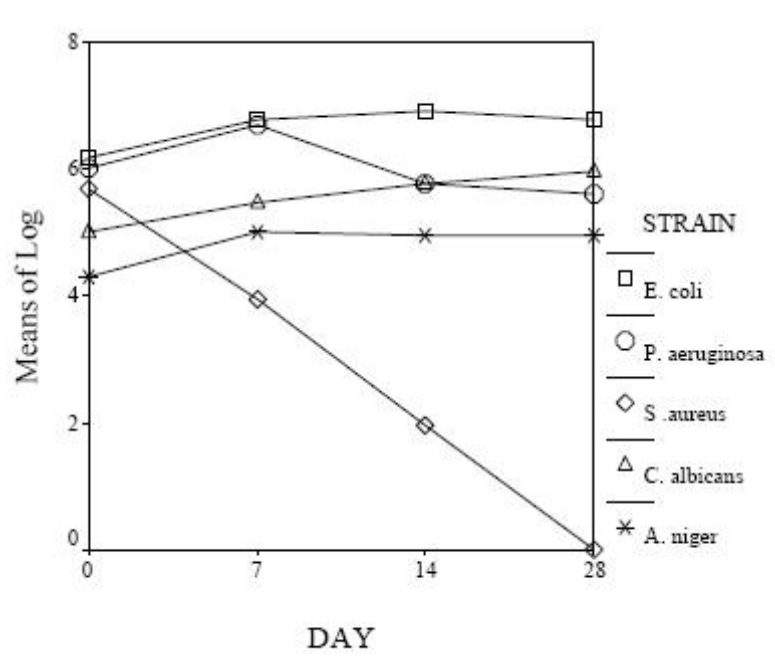

Figure 1. Means of Log of Viable Counts at Formulation $1(\mathrm{BZCl}+\mathrm{HP} \gamma-\mathrm{CD} 5 \%)$

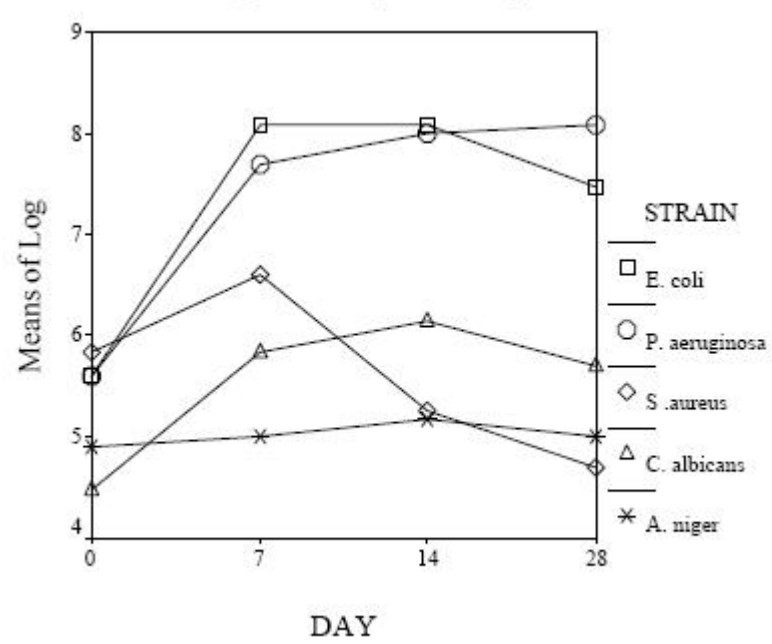

Figure 2. Means of Log of Viable Counts at Formulation 2 (MP + HP $\gamma$-CD 5\%) 


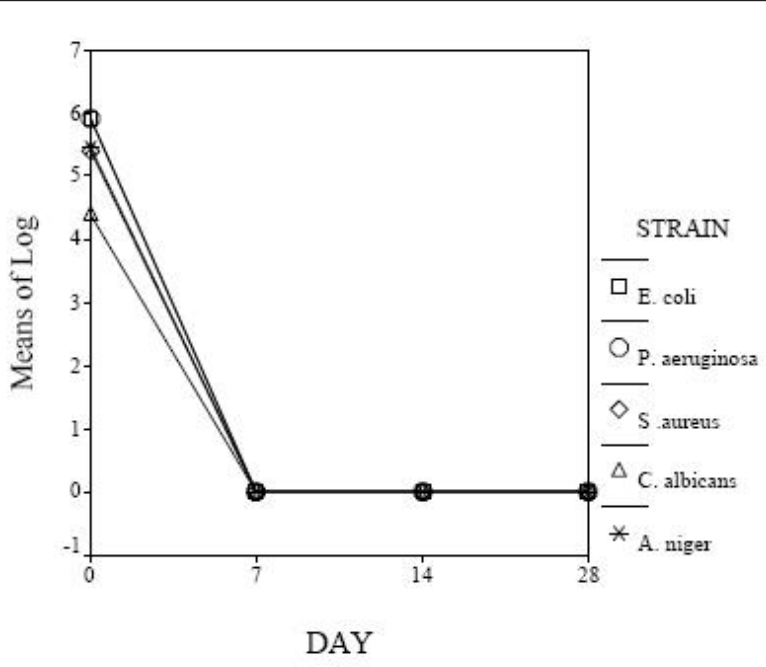

Figure 3. Means of Log of Viable Counts at Formulation 3 (EDTA + BZCl + HP $\gamma$-CD 5\%)

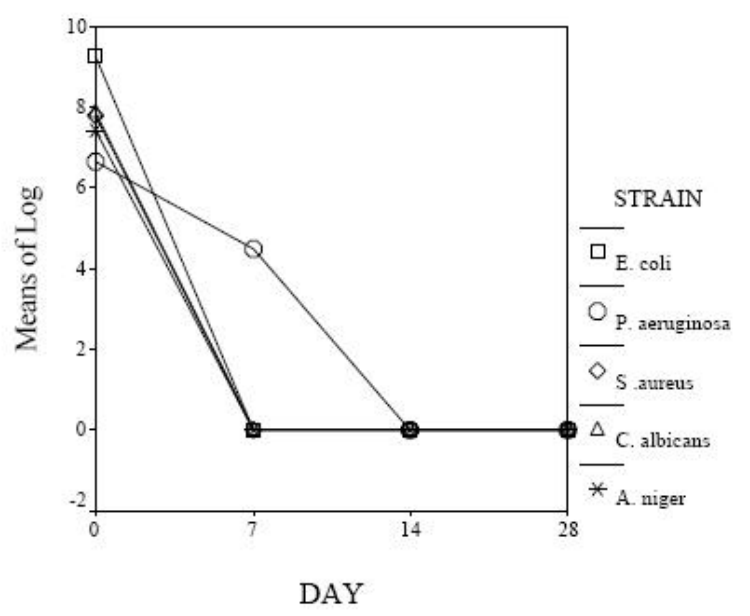

Figure 4. Means of Log of Viable Counts at Formulation 4 ( EDTA + BZCl + $\mathrm{HP} \gamma$-CD 5\% + drug)

The results also showed that preservative molecules (BZCl and EDTA) were effective in the presence of HP- $\gamma$-CD $10 \% \mathrm{w} / \mathrm{v}$, in the first week of experiment with the exception of $P$. aeruginosa, which did not show a $1 \log$ reduction in number of bacteria according to the USP (Table 6 and 7). According to the results, $\mathrm{BZCl}, \mathrm{MP}$, and their combination are not effective in the presence of HP $\gamma$-CD or SBE $\beta$-CD, even in the presence of drug molecules. The only exception was at minimum concentration of HP $\gamma$-CD (5\% w/v) which gave acceptable results according to the USP. Therefore, these interactions should be considered in drug formulation design. Overall, it seems that the best formulation for preparation of fluorometholone solution among the applied CDs concentrations, as eye drop, is HP $\gamma$-CD 5\% with $\mathrm{BZCl} 0.02 \%$ and EDTA $0.1 \%$ as preservatives.

\section{Acknowledgements}

None declared.

\section{Authors' Contribution:}

Bibi Sedigheh Fazly Bazzaz and Bizhan MalaekehNikouei designed the research plan and orginesd the study. Vahid Soheili, Kobra Mohammadian participated in all experiments, coordinated the data-analysis, and contributed to the writing the manuscript.

\section{Financial Disclosure:}

The authors declare that they have no financial interest.

\section{Funding/Support:}

This work was supported by Mashhad University of Medical Sciences, and part of it was a Pharm D thesis.

\section{References}

1. Davis ME, Brewster ME. Cyclodextrin-based pharmaceutics: past, present and future. Nat Rev Drug Discov. 2004;3(12):1023-35.

2. Del Valle EMM. Cyclodextrins and their uses: A review. Process Biochem. 2004;39(9):1033-1046.

3. Eastburn SD, Tao BY. Applications of modified cyclodextrins. Biotechnol Adv. 1994;12(2):325-39.

4. Kaur IP, Chhabra S, Aggarwal D. Role of cyclodextrins in ophthalmics. Curr Drug Deliv. 2004;1(4):351-60.

5. Loftsson T, Masson M. Cyclodextrins in topical drug formulations: Theory and practice. Int J Pharm. 2001;225(1-2):15-30.

6. Loftsson T, O' Fee R. Cyclodextrins in solid dosage forms. Business Briefing: Pharmatech. 2003:176-80.

7. Szejtli J. Introduction and General Overview of Cyclodextrin Chemistry. Chem Rev. 1998;98(5):1743-1754.

8. Loftsson T, Duchene D. Cyclodextrins and their pharmaceutical applications. Int J Pharm. 2007;329(1-2):1-11.

9. Challa R, Ahuja A, Ali J, Khar RK. Cyclodextrins in drug delivery: an updated review. AAPS PharmSciTech. 2005;6(2):E329-57.

10. Loftssona T, Järvinen T. Cyclodextrins in ophthalmic drug delivery. Adv Drug Deliv Rev.1999;36(1):59-79.

11. Shimpi S, Chauhan B, Shimpi P. Cyclodextrins: application in different routes of drug administration. Acta Pharm. 2005;55(2):139-56.

12. Stella VJ, Rajewski RA. Cyclodextrins: Their future in drug formulation and delivery. Pharm Res. 1997;14(5):556-67.

13. Hedges AR. Industrial Applications of Cyclodextrins. Chem Rev. 1998;98(5):2035-2044.

14. Stella VJ, Rao VM, Zannou EA, Zia V. Mechanisms of drug release from cyclodextrin complexes. Adv Drug Deliv Rev. 1999;36(1):3-16.

15. Chan LW, Kurup TRR, Muthaiah A, Thenmozhiyal JC. Interaction of p-hydroxybenzoic esters with beta-cyclodextrin. Int J Pharmaceut. 2000;195(1-2):71-79.

16. Lehner SJ, Muller BW, Seydel JK. Interactions between r-hydroxybenzoic acid esters and hydroxypropyl- $\beta$-cyclodextrin and their antimicrobial effect against Candida albicans. Int J Pharm. 1993;93(1-3):201-8.

17. Lehner SJ, Muller BW, Seydel JK. Effect of hydroxypropyl-beta-cyclodextrin on the antimicrobial action of preservatives.J Pharm Pharmacol. 1994;46(3):186-91.

18. Loftsson T, Stefansdottir O, Frioriksdottir H, Guomundsson O. Interactions between preservatives and 2-hydroxypropyl- $\beta$ cyclodextrin. Drug Dev Ind Pharm. 1992;18(13):1477-84.

19. Rowe RC, Sheskey PJ, Weller PJ. Handbook of pharmaceutical excipients. 2006.

20. Anonymous . USP 33 NF 28. 2010;1:48-50.

21. Astray G, Gonzalez-Barreiro C, Mejuto JC, Rial-Otero R, Simal-Gandara J. A review on the use of cyclodextrins in foods. Food Hydro- 
colloid. 2009;23(7):1631-40.

22. Brewster ME, Loftsson T. Cyclodextrins as pharmaceutical solubilizers. Adv Drug Deliv Rev. 2007;59(7):645-66.

23. Cal K, Centkowska K. Use of cyclodextrins in topical formulations: practical aspects. Eur J Pharm Biopharm. 2008;68(3):467-78.

24. Fenyvesi E', Balogh K, Ola'h E, Ba'tai B, Varga E, Molna'r M, et al. Cyclodextrins for remediation of soils contaminated with chlorinated organics. J Incl Phenom Macrocycl Chem. 2011:291-7.

25. Zhang Y, Liu Y, Liu W, Gan Y, Zhou C. Characterization of the inclusion complex of $\beta$-cyclodextrin with sorbic acid in the solid state and in aqueous solution.J Incl Phenom Macrocycl Chem. 2009;67(12):177-82.
Please cite this paper as: Malaekeh-Nikouei B, Fazly Bazzaz BS, Soheili V, Mohammadian K. Problems in Ophthalmic Drug Delivery: Evaluation of Interaction Between Preservatives and Cyclodextrins. Jundishapur J Microbiol. 2013;6(5): e6333. DOI: 10.5812/jjm.6333 